RESEARCH PAPER

\title{
The use of underground thermal gradients as technique for soil radon mitigation
}

\author{
Francisco J. Arias* and Salvador De Las Heras \\ Department of Fluid Mechanics, Polytechnic University of Catalonia, Barcelona, Spain
}

\begin{abstract}
Diffusion coefficients of radon through minerals and rocks are characterised by Arrhenius linear plots, i.e., increasing with temperature. It has been observed, for example, that rocks with a mild heating $\left(<100^{\circ} \mathrm{C}\right)$ translate into a radon release that can be enhanced 100-1,000 times than the normal release at STP (Standard Temperature Pressure (STP). Therefore, it is reasonable to think that if the soil is deliberately heated creating a thermal gradient, it could be possible, at least from a theoretical point of view, to thermally pump radon from soil because the radon atoms will escape preferentially from cold regions (low diffusion coefficient) towards hot regions (high diffusion coefficients) if a radon sink is located. In this short note, this approach for soil radon removal is investigated.
\end{abstract}

Keywords: radon mitigation; radon release; radon soil; thermal diffusion; diffusion coefficient

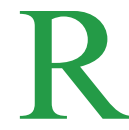

adon gas given off by soil or rocks can penetrate into housings through cracks in floors, wires, pumps, joints or gaps in foundations around pipes. It is known that radon is the number one cause of lung cancer among non-smokers, and overall, it is the second leading cause of lung cancer. According to the American Cancer Society (ACS), being exposed to radon for a long period of time can lead to lung cancer. In fact, radon gas in the air breaks down into tiny radioactive elements (radon progeny) that can lodge in the lining of the lungs, where they can give off radiation (ACS). This radiation can damage lung cells and eventually lead to lung cancer, and scientists estimate that about 20,000 lung cancer deaths per year are related to radon in the United States (1). As a result, several methods and strategies have been proposed for its mitigation/control, and an active theoretical and practical research is carried out (2). Nevertheless, according to the United States Environmental Protection Agency (EPA), today the main method used to reduce radon is sub-slab depressurisation, in which a fan and vent pipe system pulls radon from beneath the house to the outside (3).

The thermal dependence of the diffusion coefficient Since the last quarter of the past century, certain anomalies were observed in the variations of radon release from rocks concentrations during seismic activity $(4,5)$, and mounting evidence initially suggested that those variations were related with the resultant stress from the seismic episodes.
Nevertheless, radon variations were also reported during volcanic activity, and in this case, it is not certain that the stress mechanism be the dominant one (6). It was suggested, however, that the increase in the temperature of the rock due to the magma uprise was responsible for the radon release enhancement, and experimental controlled investigations appear to confirm the hypothesis (7). In any case, it has been perfectly demonstrated that radon diffusion coefficients have a temperature dependence following an Arrhenius behaviour. The last up-to-date reported data for the diffusion coefficient (2020) are shown in Fig. 1. It is seen

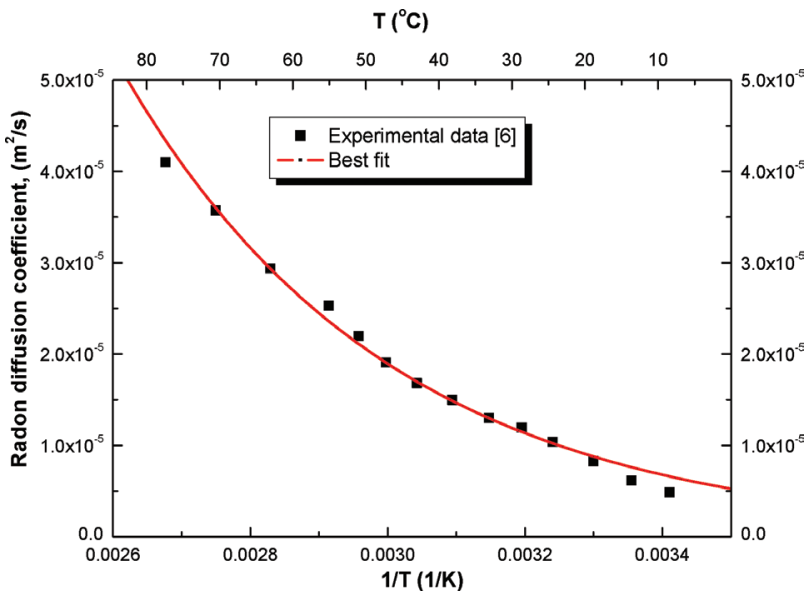

Fig. 1. Arrhenius diagram of experimental value and fitting line of the radon diffusion coefficient, from Huynh Nguyen Phong Thu et al. (8). 
that the diffusion coefficient can be enhanced up to eightfold with heating from 10 to $80^{\circ} \mathrm{C}$. This result is in agreement with previous observations performed in whole rock samples (rhyolite and alkali syenite) (7), where from a mild heating $\left(\approx 50^{\circ} \mathrm{C}\right)$ it was found that radon escapes were $100-1,000$ times higher than the radon escape at room temperature.

In this note, a first scoping calculation to assess if the thermal dependence of the mobility of radon, that is, the thermal dependence of the diffusion coefficient, may be harnessed and used as a new technologic approach for radon soil mitigation.

Figure 2 is a pictorial sketch of the core idea. In this figure, heaters are introduced underground in order to create an underground thermal gradient. The thermal source could be, for example, a simple electric generator as is shown at the right of the figure; however, the most attractive possibility is with the direct use of solar thermal insolation, for example, using a thermosyphon as shown at the left of the figure. Besides to heat the soil, the heater also serves as a sink of radon, that is, where radon is extracted constantly and radon concentration is very low. Therefore, the resulting temperature gradient (red line) translates into a concentration gradient (blue line) because the diffusion coefficient is higher as the temperature increases; the leakage of radon atoms is increased in the region where the heater is located, and then, the radon atoms are preferentially transported from cold (outer) region towards the hot (inner) region where they are removed.

For the sake of illustration, Fig. 3 shows a possible design for a heater unit. The design actually keeps resemblance with that from a fuel nuclear rod unit used in nuclear reactor technology, but where, of course, the rod is not heated by nuclear fuel but from an input of electricity or solar thermal energy. The design basically consists in a cylindrical geometry in which the heater is enclosed

\section{Electric input}

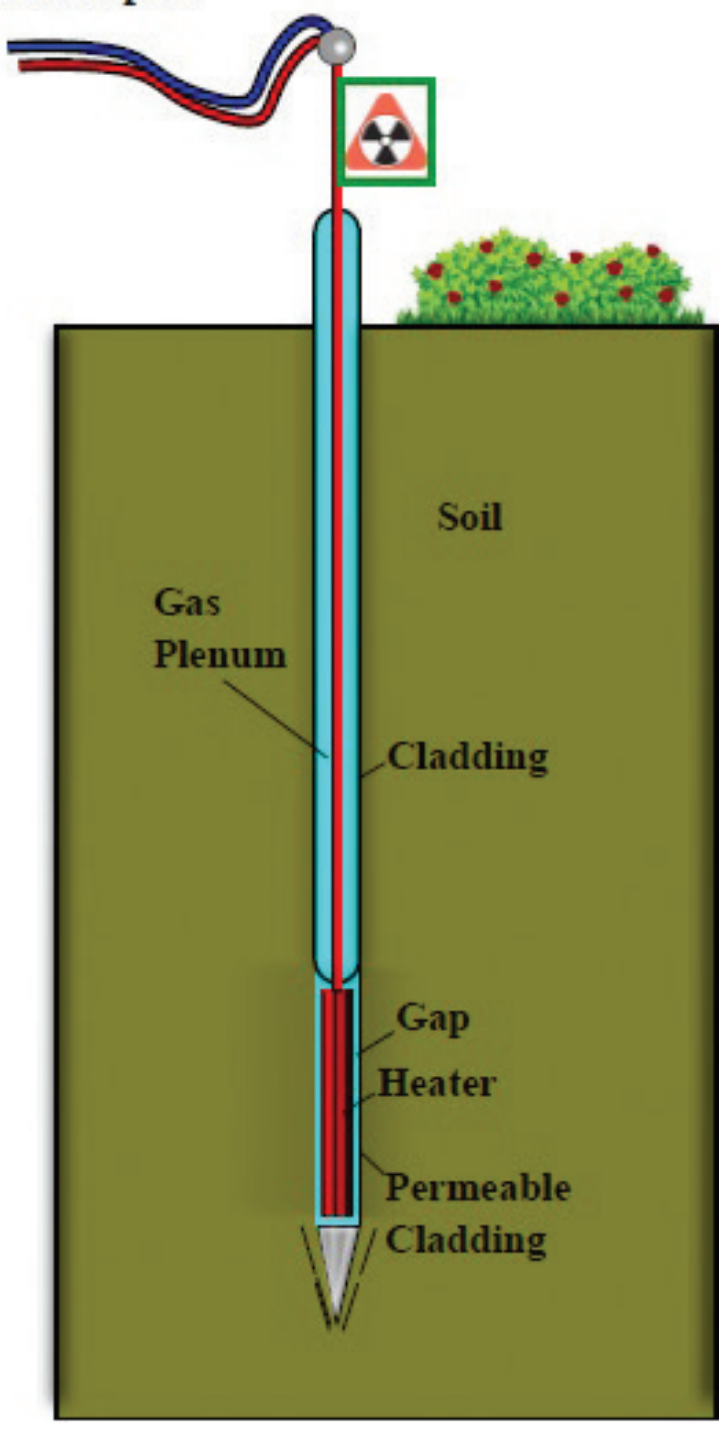

Fig. 3. A possible design for a heater unit.

\section{Thermosiphon}

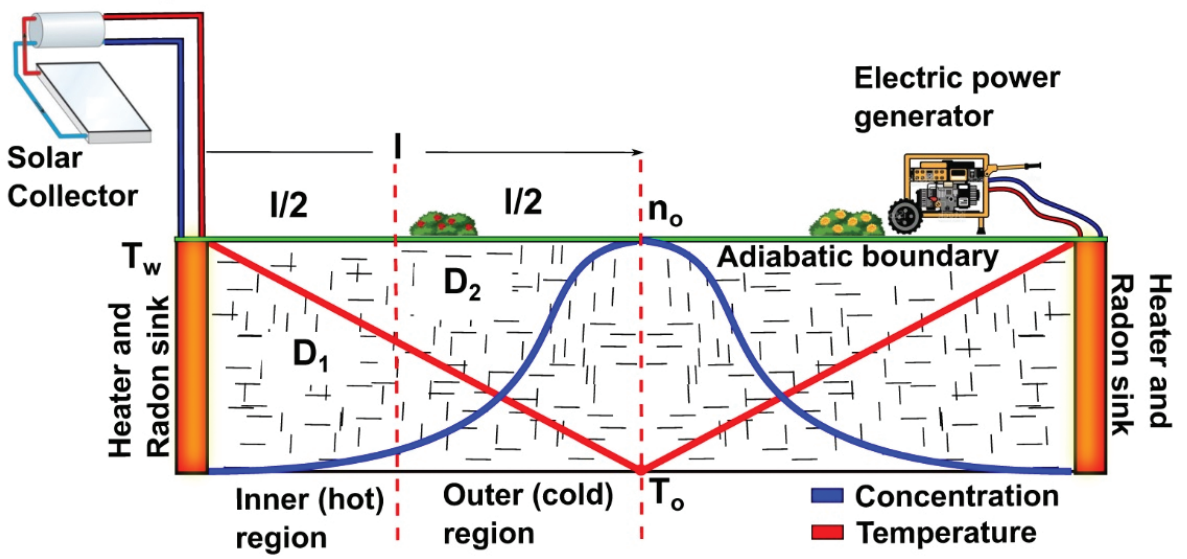

Fig. 2. Sketch of the concentration and thermal profile underground. 


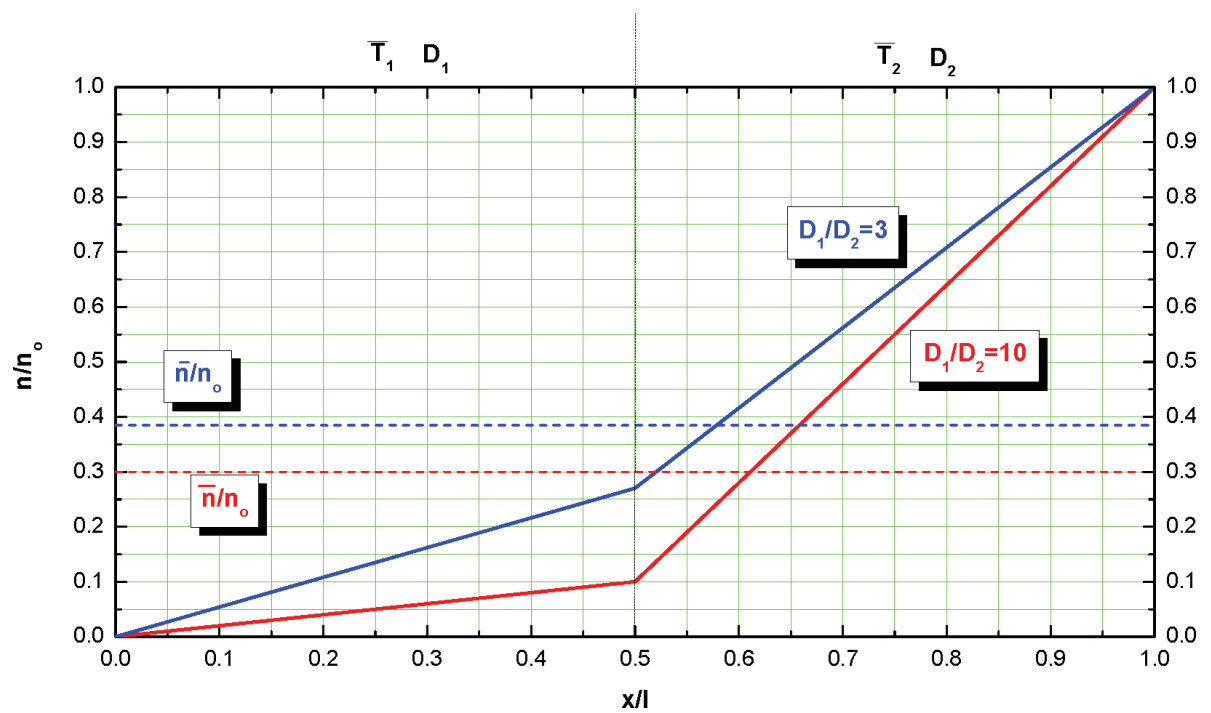

Fig. 4. Radon concentration profile using the two-group thermal model.

by a semi-permeable cladding in contact with the soil which allows the free transit of radon atoms and other gases. Between the heater and the cladding, there is a certain gap where the radon after passing through the cladding arrives. This gap allows that the gases freely travel to an upper deposit (the gas plenum) by natural convection, making that the heater besides to heat the soil also accomplishes its other function as a sink of radon.

Keeping this simple idea in mind, preliminary calculations were performed utilising a simplified two-group thermal one-dimensional model with a horizontal slab of length $l=50 \mathrm{~cm}$ in which a linear thermal gradient was imposed from the origin at $x=0$ of $T=100^{\circ} \mathrm{C}$ to the end at $x=l$ of $T=15^{\circ} \mathrm{C}, x$ being the longitudinal coordinate (9). The slab was divided into two equally spaced region: the inner hot region from $x=0$ to $x=1 / 2$; and the outer cold region from $x=l / 2$ to $x=l$. Each region was represented by an average diffusion coefficient $D_{1}$ and $D_{2}$ for the inner and outer region, respectively. The initial homogeneous concentration of radon (without thermal gradient) was $n_{0}=10^{8}$ particles per cubic meter, with a decay constant of radon atoms $\lambda_{r}=2.11 \times 10^{-6} / \mathrm{s}$, and a soil with someone arbitrary value for the thermal conductivity was $k_{t}=1 \mathrm{~W} /$ $m K$. The resulting curves are shown in Fig. 4 for two values $\frac{D_{1}}{D_{2}}=3$ and $\frac{D_{1}}{D_{2}}=10$ which can be inferred as possible by heating up to $100^{\circ} \mathrm{C}$ according to Fig. 1 .

It is seen that the average concentration of radon could be reduced to 30 to $40 \%$ for this slab with a $0.5 \mathrm{~m}$ length, that is, covering a total distance of $1 \mathrm{~m}$ between heaters. Although the reported preliminary results come from rough idealisations and are therefore not intended to typify estimates, nonetheless they give an upper limit on the feasibility for thermal radon soil removal by using underground thermal gradients and are encouraging further investigation in the subject.

\section{Conflict of interest and funding}

The authors have not received any funding or benefits from industry or elsewhere to conduct this study.

\section{References}

1. American Cancer Society. 2015. About Cancer of Unknown Primary . cancer.org | 1.800.227.2345.

2. Angell WJ. Indoor radon prevention and mitigation. In: Nriagu J, ed. Encyclopedia of environmental health. 2nd ed. Ámsterdam, Netherlands: Elsevier, 2011; p. 643-51.

3. A citizen's guide to radon: The guide to protecting yourself and your family from radon. Washington, DC: EPA; 2012. Available from: www.epa.gov/radon [cited 2016]

4. Teng TL. Some recent studies on groundwater radon content as an earthquake precursors. J Gephys Res 1980; 85: 3089-99.

5. Del Pezzo E, Gasparini P, Mantovani MSM, Martini M, Gomes YT, Capaldi G, et al. A case of correlation between Rn anomalies and seismic activity on a volcano (Vulcano Island, Southern Tirrhenian Sea). Geophys Res Lett 1984; 8: 962-5.

6. Chirkov AM. Radon as a possible criterion for predicting eruption as observed as Karymsky Volcano. Bull Volcanol 1975; 37: 126-31.

7. Gasparini P, Mantovani MSN, Ribeiro FB. Temperature dependence of radon difussion from some rocks and minerals. Bolletino Di Geofisica Ed Applicata 1984; XXVI: 101-2.

8. Phong Thu HN, Van Thang N, Hao LC (May 2020). The effects of some soil characteristics on radon emanation and diffusion. J Environ Radioact 2020; 216: 106189.

9. Arias F, De Las Heras S. (April 12, 2021). Thermal soil radon mitigation. ASME J Nucl Rad Sci. doi: 10.1115/1.4050832.

\section{*Francisco J.Arias}

Department of Fluid Mechanics,

Polytechnic University of Catalonia,

ESEIAAT C/ Colom I I, 08222 Barcelona, Spain

francisco.javier.arias@upc.edu 